\title{
High CD10 expression in lymph node metastases from surgically treated prostate cancer independently predicts early death
}

\author{
Achim Fleischmann • Carla Rocha • \\ Nikolina Saxer-Sekulic • Inti Zlobec • Guido Sauter • \\ George N. Thalmann
}

Received: 21 December 2010/Revised: 24 March 2011 /Accepted: 10 April 2011 /Published online: 3 May 2011

(C) Springer-Verlag 2011

\begin{abstract}
Patients with nodal positive prostate cancers are an important cohort with poorly defined risk factors. CD10 is a cell surface metallopeptidase that has been suggested to play a role in prostate cancer progression. CD10 expression was evaluated in 119 nodal positive prostate cancer patients using tissue microarrays constructed from primary tumors and lymph node metastases. All patients underwent radical prostatectomy and standardized extended lymphadenectomy. They had no neoadjuvant therapy and received deferred androgen deprivation. In the primary tumor, high CD10 expression was significantly associated with earlier death from disease when compared with low CD10 expression (5-year survival $73.7 \%$ vs. $91.8 \% ; p=0.043$ ). In the metastases, a high CD10 expression was significantly associated with larger total size of metastases (median 11.4 vs. $6.5 \mathrm{~mm}$; $p=$ 0.015 ), earlier death of disease (5-year survival $71.5 \%$ vs. $87.3 \% ; p=0.017)$, and death of any cause (5-year survival $70.0 \%$ vs. $87.2 \% ; p=0.001)$ when compared with low CD10 expression. CD10 expression in the metastases added independent prognostic information for overall survival $(p=0.029)$ after adjustment for Gleason score of
\end{abstract}

\footnotetext{
A. Fleischmann $(\bowtie) \cdot$ C. Rocha $\cdot$ N. Saxer-Sekulic $\cdot$ I. Zlobec Institute of Pathology, University of Bern,

CH-3010 Bern, Switzerland

e-mail: achim.fleischmann@pathology.unibe.ch

G. Sauter

Department of Pathology, University Medical Center

Hamburg-Eppendorf,

Hamburg, Germany

G. N. Thalmann

Department of Urology, University of Bern,

Bern, Switzerland
}

the primary tumor, nodal tumor burden, and resection margins. In conclusion, a high CD10 expression in prostate cancer predicts early death. This information is inherent in the primary tumors and in the lymph node metastases and might help to personalize patient management.

Keywords CD10 - Prostate cancer - Metastases · Prognosis · Tissue microarray

\section{Introduction}

Prostate cancer is the second most frequently diagnosed cancer and the sixth leading cause of cancer death in males worldwide [1]. Surgically treated lymph node positive prostate cancer patients are an important subpopulation having a similar incidence in the US like men with esophageal, gastric, or testicular cancer [2] and showing a considerable clinical, morphological, and biological heterogeneity of disease [3]. For these men, risk factors predicting fatal outcome are poorly defined.

CD10, also known as neutral endopeptidase, might be a molecular biomarker with the ability to predict death in prostate cancer. CD10 is a zinc-dependent cell surface metallopeptidase which is widely expressed in epithelial cells of various sites including kidney, breast, lung, intestine, and prostate [4]. CD10 contains a large extracellular domain known to process a variety of more than 20 neuropeptides by enzymatic cleavage [4] thus modifying the pericellular concentration of specific peptides available for receptor binding. There is evidence that peptidemediated cell growth can be suppressed or promoted by CD10 expression [5]. More recently, the effects of CD10 on growth and migration of cells have been reported which are 
independent from its enzymatic property [6]; however, the entire biological significance of CD10 is still largely unknown. The potential of CD 10 expression in prostate cancer to predict prostate-specific antigen (PSA) recurrence has been evaluated in few cohorts with conflicting results [7-11]. Importantly, survival data obtained from our cohort of 2,385 hormone naive prostate cancer patients treated by radical prostatectomy indicate early PSA failure for patients with CD10 expression [11]. This contrasts with the tumorsuppressing functions of CD10 in prostate cancer observed in functional studies [6]. Here, we tested the potential of CD10 to predict death in another well-defined cohort of surgically treated patients with lymph node positive prostate cancers.

\section{Materials and methods}

\section{Patients}

One hundred and nineteen consecutive prostate cancer patients without clinical evidence of metastases (physical examination, abdominal/pelvic computerized tomography, bone scan, and chest X-ray) but with nodal metastases upon histological examination of the lymphadenectomy specimens were studied. All patients underwent a standardized bilateral extended pelvic lymphadenectomy and radical prostatectomy as a single procedure between February 1989 and December 2006 at the Department of Urology, University of Bern. They received no neoadjuvant therapy and were followed prospectively. No adjuvant therapy, androgen deprivation in particular, was recommended until symptomatic disease progression had occurred.

Surgical technique of lymphadenectomy

Bilateral pelvic lymphadenectomy was performed in all patients as previously described [12]. Briefly, lymph node dissection was performed along the external iliac vein down to the deep circumflex iliac vein and femoral canal with the bifurcation of the common iliac artery as proximal limit of the surgical field. Next, the obturator fossa was cleared of all tissues except the nerve and vessels. Lastly, the lymphatic tissue along the medial and lateral aspect of the internal iliac artery and vein was removed. The three tissue samples from each side were submitted separately for examination.

Pathology

All prostatectomy and lymphadenectomy specimens were processed at the Institute of Pathology, University of Bern as previously described $[3,13]$. For this study, all slides were re-evaluated by experienced genitourinary pathologists (AF, CR, and NS). All Gleason patterns present in the primary tumors and lymph node metastases were tightly encircled for subsequent tissue microarray (TMA) construction.

The prostates were totally embedded. The following microscopic tumor characteristics were noted: type, Gleason score, and tumor stage. Tumor volume was estimated by multiplying the percentage of the specimen involved by cancer by the prostate volume $[3,13]$. The diameter of each lymph node metastasis was measured. The seventh edition of the TNM classification [14] was used for staging.

\section{TMA}

The TMA [15] was constructed with tissue cores of $0.6 \mathrm{~mm}$ in diameter. Each Gleason pattern present in every primary tumor and in the matched lymph node metastases as well as non-neoplastic prostate tissue (peripheral zone) is represented by one tissue core. This TMA contains 403 punch biopsy specimens from prostates (mean per patient, 3.3; range, 2-4) and 167 from lymph node metastases (mean per patient, 1.4; range, 1-3). The distribution of these samples in the prostates is as follows: normal prostate tissue $(n=119)$, Gleason pattern $3(n=101)$, Gleason pattern $4(n=112)$, and Gleason pattern $5(n=71)$. The distribution of these samples in the metastases is as follows: Gleason pattern $3(n=35)$, Gleason pattern $4(n=103)$, and Gleason pattern $5(n=29)$.

\section{Immunohistochemistry}

Freshly cut TMA sections were analyzed in 1 day in one experiment. High-temperature pretreatment of slides was done in steam with target retrieval solution, $\mathrm{pH} 9$ (Dako, Glostrup, Denmark). CD10 expression was assessed using the monoclonal antibody CD10 (clon 56C6; Novocastra, Newcastle-upon-Tyne, UK). Optimal staining was achieved at 1:50 antibody dilution. Bound primary antibodies were visualized using the Envision Plus system (Dako, Glostrup, Denmark). CD10 expression was observed on the cell membrane and in the cytoplasm (Fig. 1). Tumors could be heterogeneous in subcellular CD10 expression between different spots. Therefore, only the percentage of positive tumor cells per patient in the primary tumor and the lymph node metastases was determined. The percentage of CD10 positive tumor cells was determined for each Gleason pattern averaged over all primary tumors and nodal metastases, respectively.

Statistical analysis

CD10 expression in normal prostate, primary cancer, and metastases was compared using Kruskal-Wallis test. 
Fig. 1 CD10 expression in primary prostate cancer $(\mathbf{a}, \mathbf{b})$ and in lymph node metastases $(\mathbf{c}, \mathbf{d})$. a Few neoplastic cells with CD10 expression at the apical membrane and in the cytoplasm and immunoreactivity in the stroma. b Virtually all neoplastic cells with membranous and cytoplasmic CD10 expression. c No CD10 expression in the neoplastic cells. d Strong cytoplasmic CD10 expression in the neoplastic cells
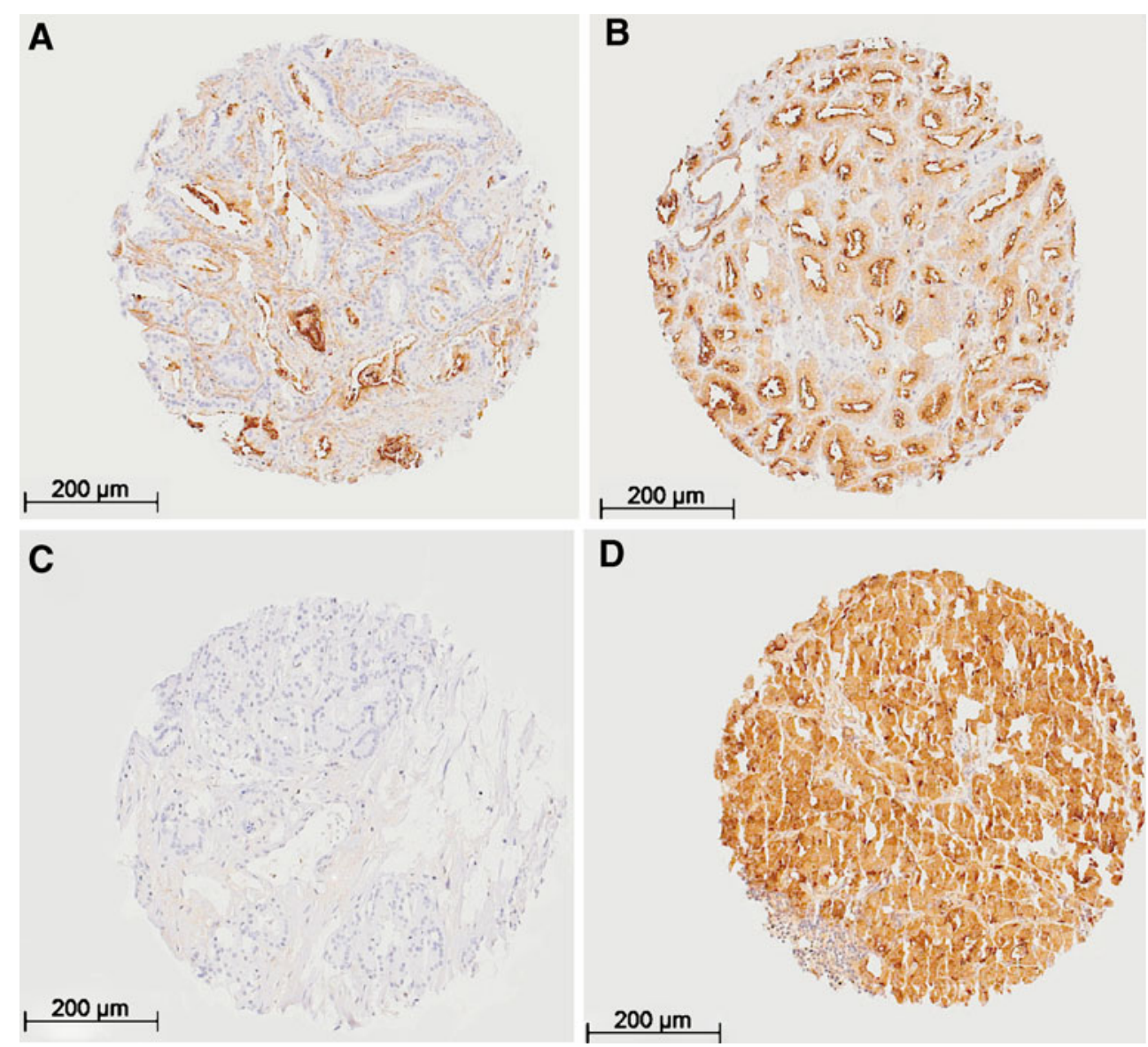

Friedman Test was used to evaluate differences between Gleason patterns 3, 4, and 5 within primary tumors and lymph node metastases, the Wilcoxon two-sample test compared expression in primary tumors and metastases stratified by the Gleason patterns. CD10 expression was correlated with (a) quantitative tumor features using the $t$ test (age) or Wilcoxon test (tumor volume, total size, and number of metastases) and (b) categorical tumor features using Fisher's exact test. Receiver-operating characteristic curves [16] were used to determine the optimal cut-off values for high (greater than $40 \%$ positive cells) and low (40\% positive cells or less) CD10 expression in both the primary tumors and lymph node metastases. Outcome was analyzed for biochemical recurrence-free, disease-specific (DSS), and overall (OS) survival defined as the intervals from surgery to the date of biochemical recurrence (PSA failure defined as values $>0.2 \mathrm{ng} / \mathrm{ml}$ ), death from prostate carcinoma, and death from any cause, respectively. Patients without event for the respective endpoints were censored at the date of last follow-up. The above time-toevents were compared between groups using log-rank test. $P$ values less than 0.05 were considered significant for all tests. After verification of the proportional hazards assumption, multiple Cox regression analysis was used for all three endpoints to determine the prognostic impact of CD10 expression in primary tumors and metastases controlling for independent risk factors determined previously in a subset of this cohort [3,13]: Gleason score of the primary tumor $(6 / 7 / 8$ vs. $9 / 10$ grouped according to similar survival curves between the clustered subsets) and lymph node tumor burden reflected by the total diameter of all nodal metastases (median value, $7.5 \mathrm{~mm}$, was used as cut-off).

\section{Results}

Characteristics of patients, prostatectomy specimens, lymphadenectomy specimens, and lymph node metastases

Baseline data of the cohort are given in Table 1.

Expression of CD10 in normal prostate, primary tumors, and lymph node metastases considering the Gleason patterns

CD10 expression was lost in primary tumors $(63.3 \%)$ and lymph node metastases $(46.9 \%)$ when compared with normal prostate glands $(90.1 \% ; p<0.001)$. No significant difference was detected between the different Gleason patterns (GP) in the primary tumors (GP3, 60.1\%; GP4, 
Table 1 Characteristics of 119 surgically treated prostate cancer patients with pelvic lymph node metastases

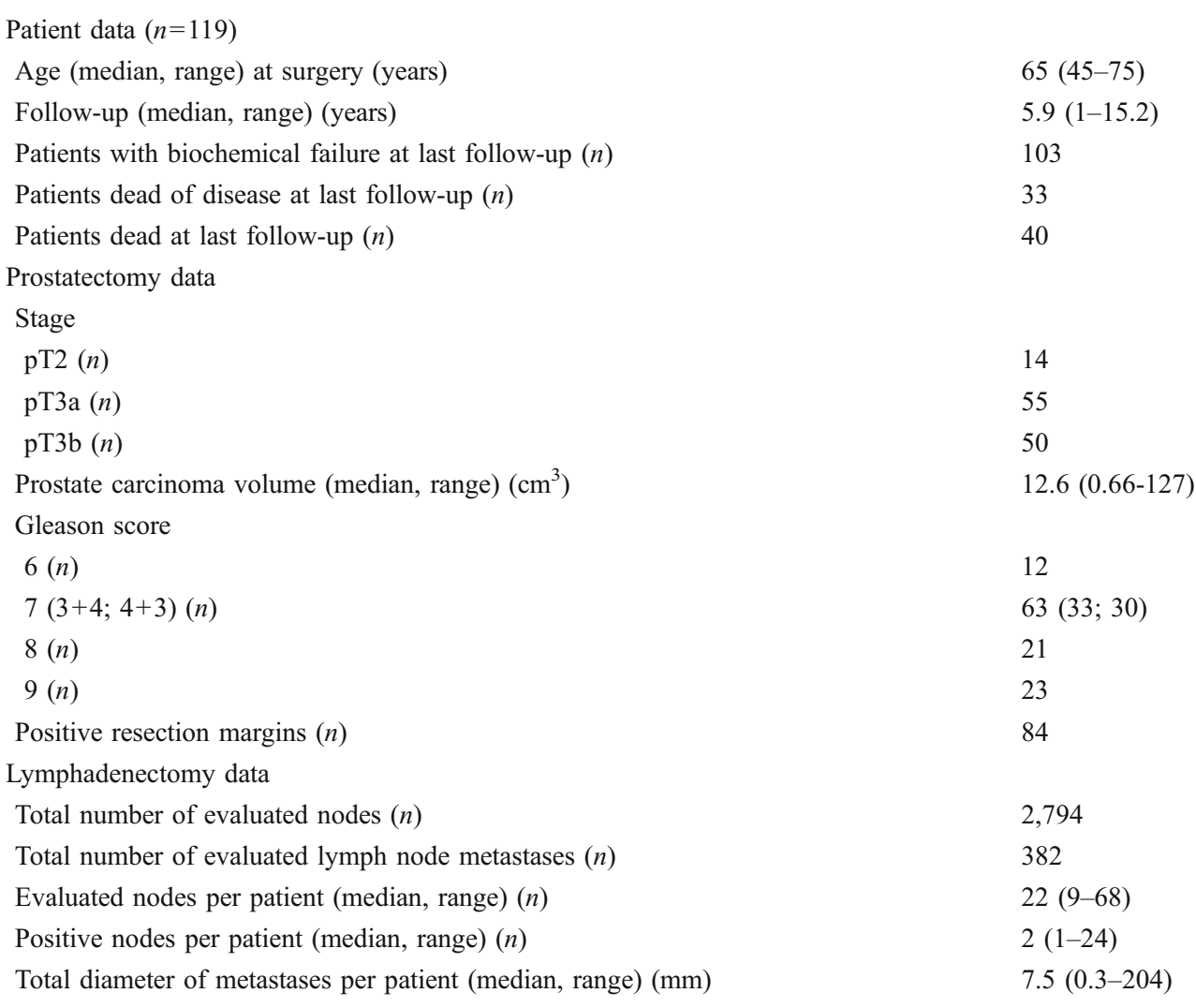

$61.0 \%$; GP5, 68.8\%; $p=0.198)$ and in the metastases (GP3, $65.3 \%$; GP4, 38.2\%; GP5, 53.7\%; $p=0.058$ ).

Correlations of CD10 expression in primary tumors and metastases with clinicopathological tumor characteristics

In the primary tumors, there was no trend for the association of CD10 with Gleason score, tumor stage, or tumor volume. In the metastases, high CD10 expression was significantly associated with larger total size of metastases (median $11.4 \mathrm{vs.}$ $6.5 \mathrm{~mm} ; p=0.015)$ but not with the median number of positive nodes (2.5 vs. $2.0 ; p=0.169)$ or lymph node Gleason score.

Univariate survival analysis according to CD10 expression

In primary prostate cancer, high CD10 expression (average percentage of CD10 positive cancer cells, 74\%) significantly predicted early death of disease ( $p=0.043$; Fig. 2) with a 5 -year DSS rate of $73.7 \%$, while patients with low CD10 expression (average percentage of CD10 positive cancer cells, $20 \%$ ) had a 5 -year DSS rate of $91.8 \%$. In the metastases, high CD10 expression (average percentage of CD10 positive cancer cells, $76 \%$ ) was a significant adverse risk factor for DSS $(p=0.017)$ and OS $(p=0.001)$ compared with low CD10 expression (average percentage of CD10 positive cancer cells, 11\%; 5-year DSS, 71.5\% vs. 87.3\%; 5-year OS, 70.0\% vs. 87.2\%; Fig. 2). The difference in PSA recurrence-free survival between patients with high and low CD10 expression was not significant (Fig. 2).

\section{Multivariate analysis}

Two separate multivariate analyses tested the prognostic impact of CD10 expression in the primary tumors and in the metastases, respectively, after adjusting for the prognostic effects of size of metastases and Gleason score of the primary tumor (Table 2). Patients with high CD10 expression in the metastases had a nearly tripled mortality (hazard ratio 2.81) compared with patients with low CD10 expression. This difference added an independent prognostic information $(p=0.029)$. The strongest prognostic factor for all endpoints was nodal tumor burden reflected by the total diameter of all metastases.

\section{Discussion}

Functional studies in single prostate cancer cell lines suggested a tumor suppressor function of CD10 [6]. However, when prostate cancer tissues were evaluated, 
Fig. 2 Survival analysis according to CD10 expression in primary prostate cancer (left) and lymph node metastases (right). Overall (first row) and diseasespecific (second row) survivals are significantly better in patients with low CD10 expression in primary tumors or metastases when compared to patients with high expression in these tumor components
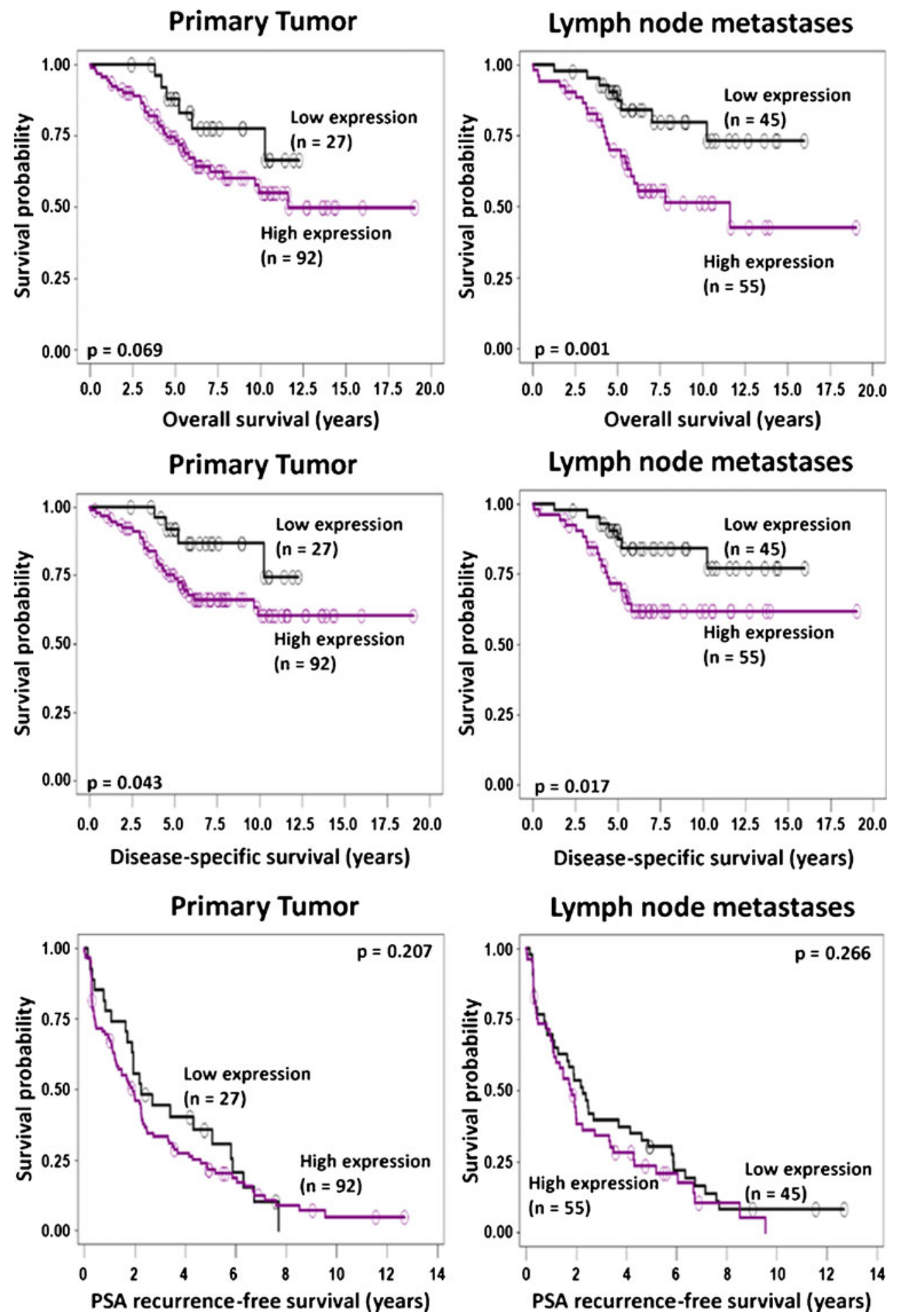

results were conflicting. Depending on the cohort, CD10 expression was considered as unfavorable [7, 11], favorable [9], or no risk factor [8, 10] for PSA recurrence after therapy. The comparability of these results, however, is limited due to large differences between the evaluated cohorts. The better outcome for CD10 positive patients reported by Osman et al. [9] was detected in a cohort of mainly African-American men with significant race- dependent differences in CD10 expression potentially impacting on results. Zellweger et al. [10] evaluated a cohort of heterogeneously treated patients including patients with hormonal therapy, which is known to suppress CD10 expression in prostate cancer via androgen responsive elements [17]. Recent data showing CD10 expression as an independent adverse risk factor for PSA recurrencefree survival in a surgically treated, hormone naive 
Table 2 Separate multivariate analyses for the prognostic impact of CD10 expression in primary prostate cancer (upper half) and lymph node metastases (lower half), respectively, after adjustment for total size of metastases, Gleason score of primary tumor, and resection margin

\begin{tabular}{|c|c|c|c|c|c|c|c|}
\hline \multirow[t]{2}{*}{ Parameter } & \multirow[t]{2}{*}{ Cut-off } & \multicolumn{2}{|l|}{ Overall survival } & \multicolumn{2}{|c|}{ Disease-specific survival } & \multicolumn{2}{|c|}{ Recurrence-free survival } \\
\hline & & $\mathrm{HR}(95 \% \mathrm{CI})$ & $P$ & HR $(95 \% \mathrm{CI})$ & $P$ & HR $(95 \% \mathrm{CI})$ & $P$ \\
\hline \multirow[t]{2}{*}{ CD10 in primary tumor } & Low & 1.0 & 0.425 & 1.0 & 0.296 & 1.0 & 0.996 \\
\hline & High & $1.43(0.6-3.5)$ & & $1.76(0.6-5.1)$ & & $1.0(0.6-1.6)$ & \\
\hline \multirow[t]{2}{*}{ Total diameter of metastases } & $<7.5 \mathrm{~mm}$ & 1.0 & $<0.001$ & 1.0 & 0.006 & 1.0 & 0.016 \\
\hline & $\geq 7.5 \mathrm{~mm}$ & $3.76(1.7-8.2)$ & & $3.45(1.4-8.3)$ & & $1.7(1.1-2.6)$ & \\
\hline \multirow[t]{2}{*}{ Gleason score } & 6 to 8 & 1.0 & 0.292 & 1.0 & 0.069 & 1.0 & 0.488 \\
\hline & 9 to 10 & $1.49(0.7-3.1)$ & & $2.05(1.0-4.4)$ & & $1.2(0.7-2.0)$ & \\
\hline \multirow[t]{2}{*}{ Resection margin } & Negative & 1.0 & 0.468 & 1.0 & 0.724 & 1.0 & 0.289 \\
\hline & Positive & $1.34(0.6-3.0)$ & & $1.17(0.5-2.7)$ & & $1.29(0.8-2.1)$ & \\
\hline \multirow[t]{2}{*}{ CD10 in metastases } & Low & 1.0 & 0.029 & 1.0 & 0.227 & 1.0 & 0.865 \\
\hline & High & $2.81(1.1-7.1)$ & & $1.92(0.7-5.5)$ & & $1.04(0.7-1.7)$ & \\
\hline \multirow[t]{2}{*}{ Total diameter of metastases } & $<7.5 \mathrm{~mm}$ & 1.0 & 0.003 & 1.0 & 0.007 & 1.0 & 0.061 \\
\hline & $\geq 7.5 \mathrm{~mm}$ & $5.18(1.7-15.3)$ & & $7.66(1.7-34.0)$ & & $1.59(1.0-2.6)$ & \\
\hline \multirow[t]{2}{*}{ Gleason score } & 6 to 8 & 1.0 & 0.532 & 1.0 & 0.075 & 1.0 & 0.789 \\
\hline & 9 to 10 & $1.31(0.6-3.1)$ & & $2.33(0.9-5.9)$ & & $1.08(0.6-1.9)$ & \\
\hline \multirow[t]{2}{*}{ Resection margin } & Negative & 1.0 & 0.462 & 1.0 & 0.825 & 1.0 & 0.133 \\
\hline & Positive & $1.45(0.5-3.9)$ & & $1.14(0.4-3.5)$ & & $1.5(0.9-2.5)$ & \\
\hline
\end{tabular}

$H R$ hazard ratio, $C I$ confidence interval

population of 2,385 prostate cancer patients [11] were in accordance with findings reported by Dall'Era et al. [7].

In this study, we extended the analysis of CD10 in prostate cancer in two respects: first, we tested the potential of CD10 to predict fatal outcome; second, the prognostic relevance was determined in a particular cohort of hormone naive, surgically treated patients with lymph node metastases. Importantly, patients with high CD10 expression, no matter if in the primary prostate cancer or in the metastasis, experience early death. High CD10 expression in the lymph node metastases added independent prognostic information and was associated with a nearly tripled risk of dying compared with patients with low CD10 expression. This is of considerable clinical interest because (a) prognostic factors are only poorly defined in surgically treated lymph node positive prostate cancer what is an important disease with a similar incidence in US American men as esophageal, gastric, or testicular cancer [2]; and (b) death is a more robust endpoint than PSA recurrence. The clinical course following PSA recurrence after radical prostatectomy is extremely variable with rapid progression in some men and an indolent course in others [18]. By contrast, death is an outcome measure of the "true" clinical course. Therefore, our data suggest that the natural history of disease in surgically treated lymph node positive prostate cancer can be accurately predicted by CD10 expression.
The prognostic potential of biomarker expression in the different tumor components of lymph node positive cancer has been compared in only few tumors [19-21] including prostate cancers [22-25]. In case of successful survival stratification, the metastasizing tumor component but not the primary tumor $[19,20,22]$ or both tumor components $[21,23,25]$ may harbor the prognostic information. In prostate cancer, CD10 expression in both tumor components, the primary cancers and the nodal metastases, predicts survival significantly. This is clinically relevant for lymph node positive patients treated by surgery because their clinical course can be predicted more precisely and adjuvant therapies might be scheduled accordingly. Moreover, risk assessment in patients with metastasized prostate cancers not qualifying for local therapies with curative intent might be improved by CD10 determination in core needle biopsies of the primary tumor. Important risk factors in this population like metastatic tumor burden and Gleason score of the primary tumor [3] can only be imprecisely determined and, therefore, the prognostic information by CD10 expression might even be superior. In this context, it is noteworthy that the tumor amount analyzed on TMAs is highly comparable to the one in the core needle biopsies so that prognostic markers identified in prostate cancer TMAs may also be relevant in the biopsy setting. 
CD10 expression in lymph node metastases of prostate cancer is positively correlated with nodal tumor burden. The pathophysiological link, however, is obscure. Conceivably, CD10 in prostate cancer metastases inactivates antiproliferative peptides thus promoting tumor growth what would be in line with the well-known enzymatic property of CD10. Alternatively, the enzymatic activity of CD10 could promote tumor growth by conversion of a propeptide into a biologically active, stimulatory form. These basic mechanisms have been suggested for ectopeptidases in general [26] and might also apply to CD10 function in prostate cancer in vivo. CD10 function depends on tissue type [7] indicated by specific tumor cell lines promoted [5] or suppressed [27] by CD10. Accordingly, CD10 expression can be a favorable [28] or an unfavorable [29] prognostic factor dependent on the cancer type.

Finally, CD10 expression in prostate cancer might also be therapeutically relevant. Newly developed CD10 inhibitors [30] may complement established therapies to counteract the unfavorable effects of CD10 on survival. Alternatively, peptide prodrugs might be applied which are enzymatically activated by CD10 expression on tumor cells. This antitumor effect has been applied successfully in a xenograft model using a CD10-overexpressing prostate cancer cell line [31].

In conclusion, $\mathrm{CD} 10$ expression in nodal positive prostate cancer predicts early death. This information may help to better predict survival individually and to personalize adjuvant therapies, thus improving patient management.

Acknowledgment This project received funding from the Bernische Krebsliga by Grant 34-904 (AF).

Conflicts of interest The authors declare that they have no conflicts of interest.

\section{References}

1. Jemal A, Bray F, Center MM, Ferlay J, Ward E, Forman D (2011) Global cancer statistics. CA Cancer J Clin 61:69-90

2. Swanson GP, Thompson IM, Basler J (2006) Current status of lymph node-positive prostate cancer: incidence and predictors of outcome. Cancer 107:439-450

3. Fleischmann A, Schobinger S, Schumacher M, Thalmann GN, Studer UE (2009) Survival in surgically treated, nodal positive prostate cancer patients is predicted by histopathological characteristics of the primary tumor and its lymph node metastases. Prostate 69:352-362

4. Erdos EG, Skidgel RA (1989) Neutral endopeptidase 24.11 (enkephalinase) and related regulators of peptide hormones. FASEB J 3:145-151

5. Carl-McGrath S, Lendeckel U, Ebert M, Rocken C (2006) Ectopeptidases in tumour biology: a review. Histol Histopathol 21:1339-1353

6. Sumitomo M, Shen R, Nanus DM (2005) Involvement of neutral endopeptidase in neoplastic progression. Biochim Biophys Acta 1751:52-59
7. Dall'Era MA, True LD, Siegel AF, Porter MP, Sherertz TM, Liu AY (2007) Differential expression of CD10 in prostate cancer and its clinical implication. BMC Urol 7:3

8. Freedland SJ, Seligson DB, Liu AY et al (2003) Loss of CD10 (neutral endopeptidase) is a frequent and early event in human prostate cancer. Prostate 55:71-80

9. Osman I, Yee H, Taneja SS et al (2004) Neutral endopeptidase protein expression and prognosis in localized prostate cancer. Clin Cancer Res 10:4096-4100

10. Zellweger T, Ninck C, Mirlacher M et al (2003) Tissue microarray analysis reveals prognostic significance of syndecan-1 expression in prostate cancer. Prostate 55:20-29

11. Fleischmann A, Schlomm T, Huland $\mathrm{H}$ et al (2008) Distinct subcellular expression patterns of neutral endopeptidase (CD10) in prostate cancer predict diverging clinical courses in surgically treated patients. Clin Cancer Res 14:7838-7842

12. Bader P, Burkhard FC, Markwalder R, Studer UE (2003) Disease progression and survival of patients with positive lymph nodes after radical prostatectomy. Is there a chance of cure? J Urol 169:849-854

13. Fleischmann A, Schobinger S, Markwalder R et al (2008) Prognostic factors in lymph node metastases of prostatic cancer patients: the size of the metastases but not extranodal extension independently predicts survival. Histopathology 53:468-475

14. Sobin LH, Gospodarowicz MK, Wittekind C (eds) (2009) TNM classification of malignant tumours, 7th edn. Wiley, New York

15. Kononen J, Bubendorf L, Kallioniemi A et al (1998) Tissue microarrays for high-throughput molecular profiling of tumor specimens. Nat Med 4:844-847

16. Soreide K (2009) Receiver-operating characteristic curve analysis in diagnostic, prognostic and predictive biomarker research. J Clin Pathol 62:1-5

17. Zheng R, Shen R, Goodman OB Jr, Nanus DM (2006) Multiple androgen response elements cooperate in androgen regulated activity of the type 1 neutral endopeptidase promoter. Mol Cell Endocrinol 259:10-21

18. Freedland SJ, Humphreys EB, Mangold LA et al (2005) Risk of prostate cancer-specific mortality following biochemical recurrence after radical prostatectomy. JAMA 294:433-439

19. Cabanillas R, Rodrigo JP, Astudillo A, Dominguez F, Suarez C, Chiara MD (2007) P53 expression in squamous cell carcinomas of the supraglottic larynx and its lymph node metastases: new results for an old question. Cancer 109:1791-1798

20. Harigopal M, Berger AJ, Camp RL, Rimm DL, Kluger HM (2005) Automated quantitative analysis of E-cadherin expression in lymph node metastases is predictive of survival in invasive ductal breast cancer. Clin Cancer Res 11:4083-4089

21. Wang YF, Chen JY, Chang SY et al (2008) Nm23-H1 expression of metastatic tumors in the lymph nodes is a prognostic indicator of oral squamous cell carcinoma. Int J Cancer 122:377-386

22. Cheng L, Leibovich BC, Bergstralh EJ et al (1999) p53 alteration in regional lymph node metastases from prostate carcinoma: a marker for progression? Cancer 85:2455-2459

23. Masuda M, Takano Y, Iki M et al (1998) Prognostic significance of $\mathrm{Ki}-67, \mathrm{p} 53$, and $\mathrm{Bcl}-2$ expression in prostate cancer patients with lymph node metastases: a retrospective immunohistochemical analysis. Pathol Int 48:41-46

24. Bostwick DG, Qian J, Pacelli A et al (2002) Neuroendocrine expression in node positive prostate cancer: correlation with systemic progression and patient survival. J Urol 168:12041211

25. Quek ML, Daneshmand S, Rodrigo S et al (2006) Prognostic significance of neuroendocrine expression in lymph node-positive prostate cancer. Urology 67:1247-1252

26. Nanus DM (2003) Of peptides and peptidases: the role of cell surface peptidases in cancer. Clin Cancer Res 9:6307-6309 
27. Erhuma M, Kobel M, Mustafa T et al (2007) Expression of neutral endopeptidase (NEP/CD10) on pancreatic tumor cell lines, pancreatitis and pancreatic tumor tissues. Int $\mathrm{J}$ Cancer 120:2393-2400

28. Mondada D, Bosman FT, Fontolliet C, Seelentag WK (2006) Elevated hepatocyte paraffin 1 and neprilysin expression in hepatocellular carcinoma are correlated with longer survival. Virchows Arch 448:35-45

29. Tajima Y, Yamazaki K, Nishino N et al (2004) Gastric and intestinal phenotypic marker expression in gastric carcinomas and recurrence pattern after surgery-immunohistochemical analysis of 213 lesions. Br J Cancer 91:1342-1348

30. Battistini B, Daull P, Jeng AY (2005) CGS 35601, a triple inhibitor of angiotensin converting enzyme, neutral endopeptidase and endothelin converting enzyme. Cardiovasc Drug Rev 23:317-330

31. Pan C, Cardarelli PM, Nieder MH et al (2003) CD10 is a key enzyme involved in the activation of tumor-activated peptide prodrug CPI-0004Na and novel analogues: implications for the design of novel peptide prodrugs for the therapy of $\mathrm{CD} 10+$ tumors. Cancer Res 63:5526-5531 